\title{
Digital Image Watermarking using Fuzzy Logic approach based on DWT and SVD
}

\author{
T.Sridevi \\ Associate Professor \\ CBIT \\ Hyderabad,India
}

\author{
S Sameena Fatima,Ph.D \\ Professor, OU, \\ Hyderabad, India
}

\begin{abstract}
Digital image watermarking is implemented using fuzzy logic approach based on transform domain. Fuzzy logic is an approach based on "degrees of truth" rather than usual "true or false" i.e., Boolean logic. In this paper, we use a reference image instead of original image. To get a reference image Directive contrast and Threshold value are calculated for middle and high frequency bands. A transform domain with the help of fuzzy logic system is used to embed the watermark in the reference image. Here fuzzy logic is used to find the alpha value that is used to embed the watermark. Watermark embedding is based on DWT and Singular Value Decomposition (SVD). After decomposing the cover image into four bands, SVD is applied to one of the frequency bands, and embed the same watermark data by modifying the singular values. Attacks are also performed on watermarked image. Quantitative measures like PSNR, NCC are also calculated to test the watermarked image and also extracted watermarks with and without attacks
\end{abstract}

\section{General Terms}

Watermarking, Security, Algorithms.

\section{Keywords}

Digital watermarking, Fuzzy logic, Original image, Directive contrast, Threshold, Reference image, DWT (Discrete Wavelet Transformation), SVD (Singular Value Decomposition).

\section{INTRODUCTION}

The development of digital technology brings a demanding of digital data protection. Among different protection techniques, digital watermarking appears as an effective solution. Digital image watermarking is a technique used to hide the information in the image for copy right protection. The main issues to be considered in the watermarking are imperceptible, robustness, blindness and capacity. Imperceptible is required so that a watermark may remain invisible in order to maintain its secrecy i.e., the quality of image should not be destroyed. Robustness means that a watermark is able to effectively resist on common digital image attacks, such as low pass filtering, cropping, and rotation. Capacity is defined as a technique used to embed the maximum watermark.

The two techniques to embed watermark are spatial domain techniques [6,7] and transform domain techniques [810]. Due to poor fidelity and robustness spatial domain is not that suitable for watermark embedding. Watermark in frequency domain can be embedded in any transforms like DCT(Discrete domain transform) ,DWT(Discrete wavelet transform) etc.
A wavelet based watermarking exploiting HVS (human visual system) was proposed by Barni et al. [11]. Chaotic logistic map based watermarking was proposed by Dawei et al. [12] .This watermarking algorithm illustrated robustness against geometric attacks and susceptible to common attacks .

Liu et al. [3] proposed SVD based watermarking, in which singular values of cover image are modified by adding watermark and extraction is reverse process.

Sridevi et al[5] presented a watermarking method based on DWT and Singular Value Decomposition (SVD). Watermark is embedded by modifying the singular values.

A robust reference watermarking method is proposed by Joo et al. [4] . Watermark is embedded at locations where the variation between the lower bands of both cover image and reference image is minute.

Liu et al. [3] tailored Joo's scheme. Reference image is constructed where all the high frequency components are initialized to zero. A location is computed using some equation which is based on the variation between cover image and reference image. The value of the equation decides the watermark embedding.

A new reference watermarking technique proposed by Bhatnagar etal[2].the DC (directive contrast )for each sub band is calculated and Reference image is constructed by setting 1 the wavelet coefficients to zero which have DC less than a given threshold. In the modified singular values watermark is embedded in the reference image.

David J. Coumou et al. [1] projected a watermarking technique where a fuzzy inference system is used to compute the positions of the watermark which will achive both robustness and fidelity

This paper mainly focuses on DWT and SVD. Fuzzy logic is applied for finding the pixel adjustment, to embed watermark for achieving fidelity. Here new reference image is formed using directive contrast and threshold. The difference between cover image and reference image is found...Based on the difference fuzzy logic is applied to find the pixel adjustment.

\section{PRELIMINARIES}

The Preliminaries are discussed below.

\subsection{Discrete Wavelet Transform}

The Discrete Wavelet Transform (DWT)(fig 1) is achieved by filtering the signal through a series of digital filters at different scales.An outstanding space and frequency energy compaction is provided by wavelet techniques and hence DWT has received an interest in several signal and image processing applications. 
Fig 1:Discrete Wavelet Transformation

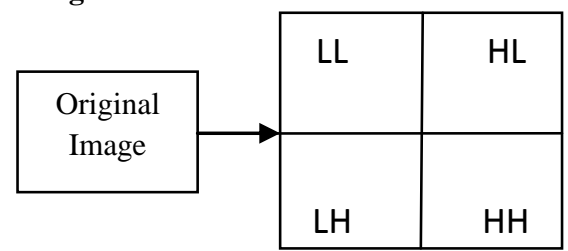

\subsection{SVD}

The singular value decomposition of a rectangular matrix A is a decomposition of the form

$$
\mathrm{A}=\mathrm{UDV}^{\mathrm{T}}
$$

Where $\mathrm{A}$ is an $\mathrm{m} * \mathrm{n}$ matrix, $\mathrm{U}, \mathrm{V}$ are ortho-normal matrices. $\mathrm{D}$ is a diagonal matrix comprised of singular values of $\mathrm{A}$. $\mathrm{D}=$ $\operatorname{diag}(\sigma 1, \sigma 2, \sigma 3 \ldots)$

It is important to note that each singular value specifies the luminance of an image layer while the corresponding pair of singular vectors specifies the geometry of the image layer. In SVD-based watermarking, several approaches are possible. A common approach is to apply SVD to the whole cover image. [5]

\subsection{FUZZY LOGIC}

The principle of fuzzy logic reaches the human approach in the sense that the variables treated are not binary but of variable linguistic relatives of human language as high contrast, a lot more clearly, very textured, very homogeneous etc. Moreover, these linguistic variables are processed with rules that refer to some knowledge of system. The fuzzifier and the defuzzifier have the role of converting external information in fuzzy quantities and vice versa.

FIS as in fig 2 consists of four function blocks. They are

1. Fuzzifier: It transfers the crisp input to fuzzy sets

2. Knowledge Base: It mainly consists of database and rule base. The data base defines the membership functions of the linguistic variables. The rule base consists of a set of IFTHEN rules that can be given by a human expert or also can be extracted from the linguistic description of the data.

3. Inference Engine: It is a general control mechanism that exploits the fuzzy rules and the fuzzy sets defined in the Knowledge Base in order to reach certain conclusion.

4. De-Fuzzifier: It transfers the fuzzy sets into crisp outputs.

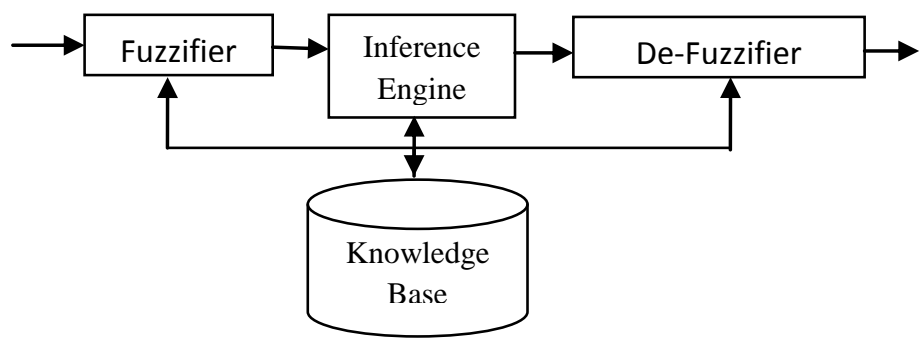

Fig 2:Fuzzy Inference system[3]

\section{PROPOSED METHOD}

The Proposed method for embedding watermark in the cover image is discussed below

\subsection{Fuzzy Rules}

In this paper, fuzzy rules are applied on the intensities of pixel values as in [1].

1. Inputs to Fuzzy are collected from the sub images that connect to each corner of sub image that is being manipulated to embed the watermark feature.

2. Fuzzifier identifies the four input sub images with the labels par_1, par_2, par_3, par_4. (The mean intensity of the pixel elements contained in each input sub-image is calculated as the inputs to Fuzzy)

3. Each input is composed of three membership functions that are based on the gray scale of Black, Gray, and White.

4. The insertion sub image is manipulated sub image for the watermark feature.

5. The output of Fuzzy is pixel adjustment (PixAdj) applied uniformly to watermark and is based on the Three output membership function Minimum (min), Medium (med), Maximum (max).

6. These rules correlate the amount of adjustment applied to the pixels in watermark with the intensity of the pixels contained in par_1, par_2, par_3 and par_4.

The fuzzy membership functions are shown in fig 3

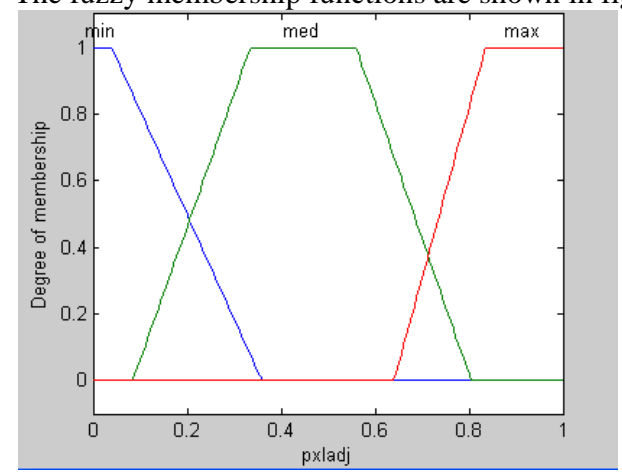

Fig 3:Fuzzy membership functions

\subsection{Procedure to find embedding strength}

1. Perform 1-level dwt to original image.

2. Take LL-band and apply one level dwt to LL-band.

3. Calculate directive contrast and threshold for middle and high frequency bands [2].

4. Initialize high frequency components to zero whose directive contrast are less than the Threshold [2].

5. Perform inverse dwt and obtain reference image.

6. Replace reference image in the selected LL band (step2) and apply inverse dwt.

7. A new reference image is obtained.

8. Perform 1 level dwt to original image and this new reference image

9. Select LL BANDS of both the images and calculate difference between them

Use fuzzy logic as shown in fig 4 to build the rules for pixel adjustment. 


\subsection{Watermark embedding[5]}

The steps for embedding the Algorithm is

1. Using DWT, decompose the cover image A into 4 sub bands: LL, HL, LH, and HH.

2. Apply SVD to HL sub band cover image: values of $\mathrm{D}_{\mathrm{a}}$.

$$
\mathrm{A}=\mathrm{U}_{\mathrm{a}} \mathrm{D}_{\mathrm{a}} \mathrm{V}_{\mathrm{a}}, \quad \sigma_{\mathrm{i}}, \mathrm{i}=1, \ldots, \mathrm{n} \text { are the singular }
$$

3. Using DWT, decompose the watermark image $\mathrm{W}$ into 4 sub bands: LL, HL, LH, and HH.

4. Apply SVD to the visual watermark:

$\mathrm{W}=\mathrm{U}_{\mathrm{W}} \mathrm{D}_{\mathrm{W}} \mathrm{V}_{\mathrm{W}}^{\mathrm{T}}$, where $\sigma_{\mathrm{wi}}, \mathrm{i}=1, \ldots, \mathrm{n}$ are the singular values of $\mathrm{D}_{\mathrm{W}}$.

5. Modify the singular values of the cover image in each sub band with singular values of visual watermark:The value of pixel adjustment $(\alpha)$ from the fuzzy logic.

$$
\sigma^{*}=\sigma_{\mathrm{i}}+\alpha \sigma_{\mathrm{wi}}
$$

6. Obtain the 4 sets of modified DWT coefficients

$$
\mathrm{A}_{\mathrm{w}}=\mathrm{U} \sigma^{*} \mathrm{~V}^{\mathrm{T}}
$$

\subsection{Watermark extraction}

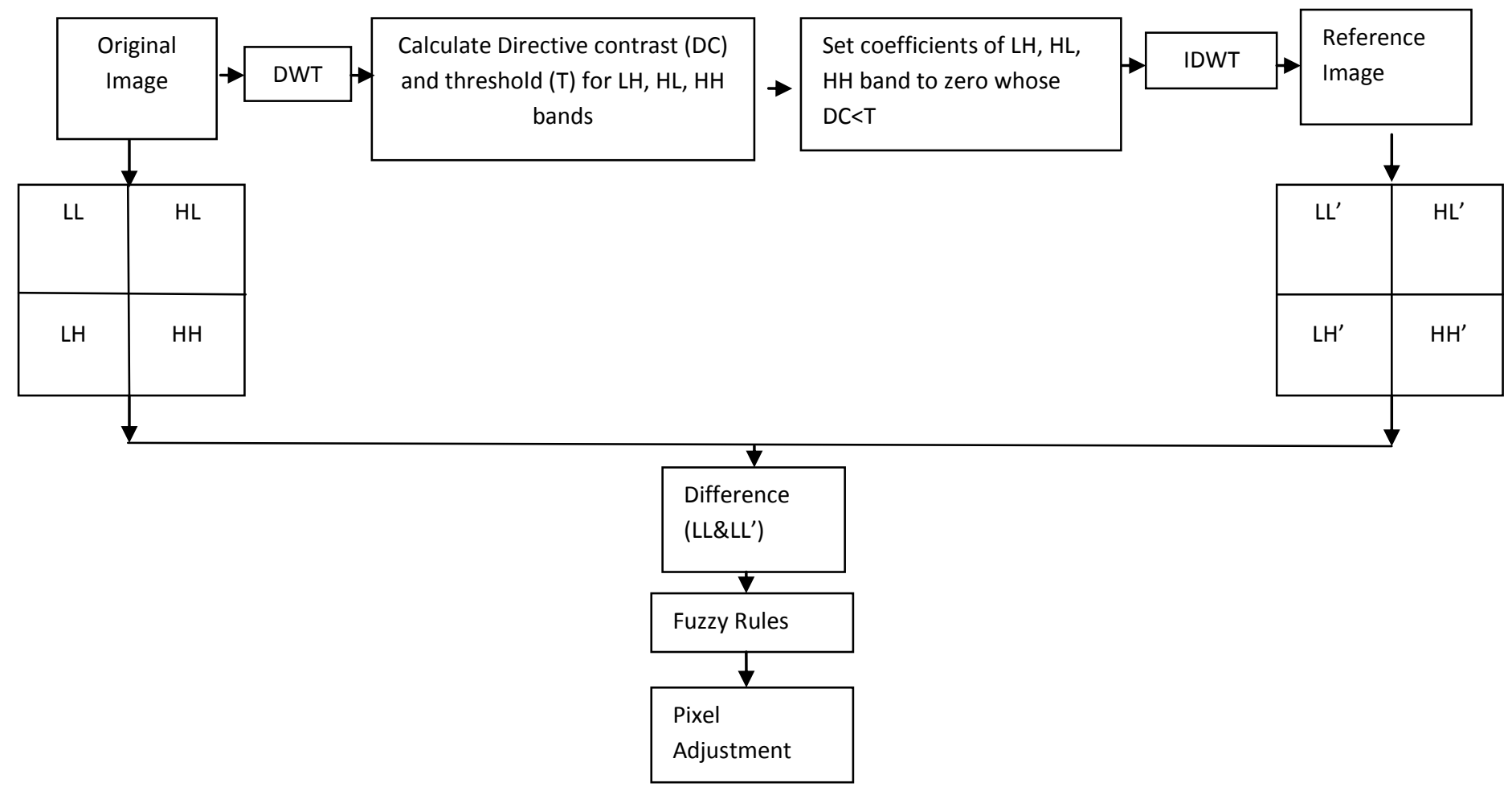

Using DWT, decompose the watermarked (and possibly attacked) cover image $A_{w}$ into 4 sub band s: LL, HL, LH, and HH.

1. Apply SVD to sub band of watermarked image $\mathrm{A}_{\mathrm{w}}=\mathrm{U} \sigma^{*} \mathrm{~V}^{\mathrm{T}}$

2. Extract the singular values from one sub band. The value of pixel adjustment $(\alpha)$ from the fuzzy logic

$$
\sigma_{\mathrm{wi}}=\left(\sigma_{\mathrm{i}}{ }^{*}-\sigma_{\mathrm{i}}\right) / \alpha
$$

3. Construct the visual watermark using the singular vector

$$
\mathrm{W}=\mathrm{U}_{\mathrm{w}} \sigma_{\mathrm{w}} \mathrm{V}_{\mathrm{w}}{ }^{\mathrm{T}}
$$

4. We computed the singular values in the sub bands

\section{RESULTS}

In order to explore the performance of the proposed watermarking algorithm, MATLAB platform is used and a number of experiments are performed on different images. The sample images are shown in Fig 5(a-h) below. The PSNR values are shown in Fig 6.To test the robustness of the algorithm PSNR values are evaluated between original images and modified images. Except noise attack all the remaining attacks are resistant to the attacks. The embedding strength is based on the HVS properties of the images.

Fig 4: Procedure for finding embedding Pixel Adjustment 


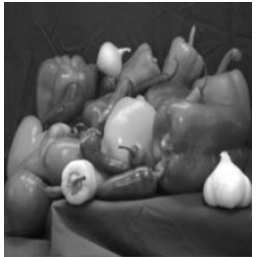

a. Original image

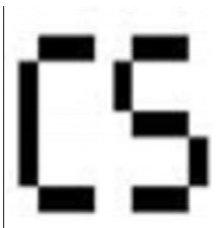

e. Extracted watermark $(\mathrm{PSNR}=$

$37.6859 \mathrm{~dB}$ and $\mathrm{NCC}=0.999$ )

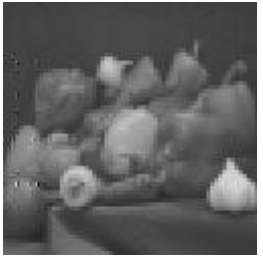

b. Reference image

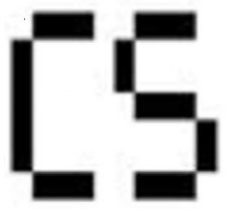

f. Extracted watermark after rotation $(\mathrm{PSNR}=46.7970 \mathrm{~dB})$ B) g. Extracted watermark after histogram equalization $(\mathrm{PSNR}=41.75 \mathrm{~d}$

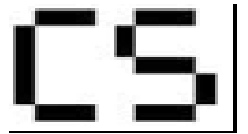

d. Original watermark

c. Watermarked image $(\mathrm{PSNR}=42.3556 \mathrm{dBan}$ $\mathrm{d} \mathrm{NCC}=0.999$ )
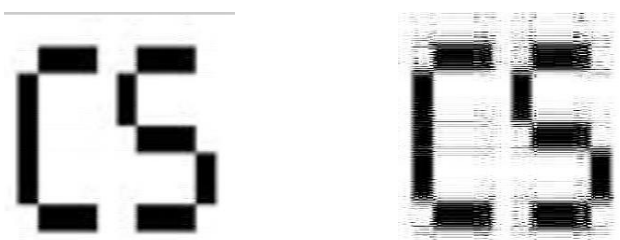

h. Extracted watermark after guassian noise $(\mathrm{PSNR}=11.2648 \mathrm{~dB})$

Fig 5(a-h):Sample image and extracted images

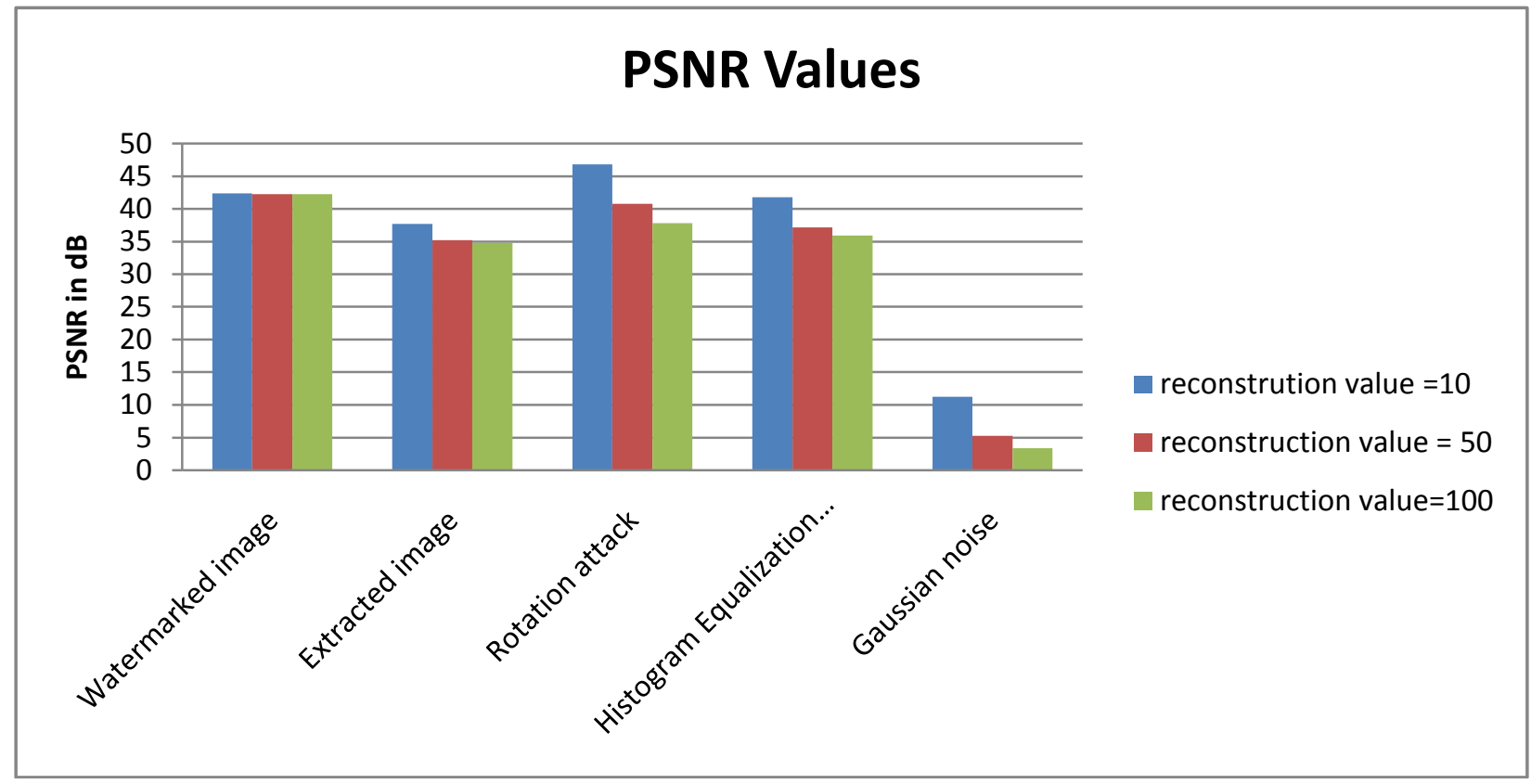

Fig 6: PSNR values for different reconstruction values 


\section{CONCLUSION}

A robust watermarking using fuzzy logic approach based on DWT and SVD algorithm was implemented .SVD is a convenient tool for Watermarking in Discrete Wavelet Transform domain. The fuzzy logic decided how much of watermark has to be added to the cover image, which is based on the image properties .The Singular Values (k) increases, the value of PSNR is not varying much. Different attacks are implemented .The noise attack is not resistant to the algorithm The PSNR values of retrieved watermark is very low but the visual quality is good. This investigation may be further extended to multiple levels of DWT decomposition and different watermark representations .In SVD watermarking, singular values are embedded into singular values, instead of singular values any other vector that represents some information may be used.

\section{REFERENCES}

[1]. David J. Coumou, Athimoottil Mathew, A fuzzy logic approach to digital image watermarking, Rochester Institute of technology, 2000.

[2]. Bhatnagar, Gaurav, and Balasubramanian Raman. "A new robust reference watermarking scheme based on DWT-SVD." Computer Standards \& Interfaces31.5 (2009): 1002-1013.

[3]. J.L. Liu, D.C. Lou, M.C. Chang, H.K. Tso, A robust watermarking scheme using self reference image, Computer standards and interfaces 28 (2006) 356-367.

[4]. S.Joo, Y. Suh, J. Shin, H. Kitkuchi, A new robust watermarking embedding into wavelet DC components, ETRI Journal 24 (5) (2002) 401-404.
[5]. T.Sridevi etal," Secure Watermarking based on svd and wavelets", ICGST International Journal on graphics,vision and Image processing, Volume 10,IssueV,Dcember 2010,pp 63-69.

[6]. A.G. Bors, I. Pitas, "Image watermarking using DCT domain constraints", Proceedings of IEEE International Conference on Image Processing, vol. 3, 1996, pp. 231234.

[7]. R.G.V. Schyndle, A.Z. Tirkel, C.F. Osbrone, "A digital watermark", Proceedings of IEEE International Conference on Image Processing, vol. 2, 1994, pp. 8690.

[8]. D. Kundur, D. Hatzinakos, Towards robust logo watermarking using multiresolution image fusion, IEEE Transcations on Multimedia 6 (2004) 185- 197.

[9]. J. Ohnishi, K. Matsui, "Embedding a seal in to a picture under orthogonal wavelet Transform", Proceedings of IEEE International Conference on Multimedia and Computing system, 1996, pp. 514-521, IEEE, Hiroshima, Japan.

[10].P. Meerwald, A. Uhl, "A survey on wavelet domain watermarking algorithms", Proceedings of SPIE, Electronic Imaging, Security and Watermarking of Multimedia Contents III, vol. 4314, 2001, pp. 505-516, SPIE, CA, USA.

[11].M. Barni, F. Bartolini, A. Piva, Improved wavelet based watermarking through pixel wise masking, IEEE Transcations on Image Processing 10 (2001) 783-791.

[12].Z. Dawei, C. Guanrong, L. Wenbo, A chaos based robust wavelet domain watermarking algorithm, Chaos, Solitons, and Fractals 22 (2004) 47-54. 